\title{
Probability of Early Retirement Among Emergency Physicians
}

\author{
Jaemyeong Shin', Yun Jeong Kim'1, Jong Kun Kim', Dong Eun Lee', Sungbae Moon', Jae Young Choe', Won Kee Lee², \\ Hyung Min Lee ${ }^{3}$, Kwang Hyun $\mathrm{Cho}^{4}$ \\ ${ }^{1}$ Department of Emergency Medicine, Kyungpook National University School of Medicine, Daegu, Korea; ${ }^{2}$ Medical Research Collaboration Center in \\ Kyungpook National University Hospital \& Kyungpook National University School of Medicine, Daegu, Korea; ${ }^{3}$ Department of Emergency Medicine, \\ Korea University Guro Hospital, Seoul, Korea; ${ }^{4}$ Department of Emergency Medicine, Nowon Eulji Medical Center, Eulji University, Seoul, Korea
}

Objectives: Early retirement occurs when one's job satisfaction suffers due to employment mismatch resulting from factors such as inadequate compensation. Medical doctors report high levels of job stress and burnout relative to other professionals. These levels are highest among emergency physicians (EPs), and despite general improvements in their working conditions, early retirement continues to become more common in this population. The purpose of this study was to identify the factors influencing EPs intention to retire early and to develop a probability equation for its prediction.

Methods: A secondary analysis of data from the 2015 Korean Society of Emergency Physicians Survey was performed. The variables potentially influencing early retirement were organized into personal characteristics, extrinsic factors, and intrinsic factors. Logistic regression analysis was performed to identify risk factors and to develop a probability equation; these findings were then arranged in a nomogram.

Results: Of the 377 survey respondents included in the analysis, $48.0 \%$ intended to retire early. Risk factors for early retirement included level of satisfaction with the specialty and its outlook, slanderous reviews, emergency room safety, health status, workload intensity, age, and hospital type. Intrinsic factors (i.e., slanderous reviews and satisfaction with the specialty and its outlook) had a stronger influence on early retirement than did extrinsic factors.

Conclusions: To promote career longevity among EPs, it is vital to improve emergency room safety and workload intensity, to enhance medical professionalism through a stronger vision of emergency medicine, and to strengthen the patient-doctor relationship.

Key words: Emergency physicians, Intention, Nomograms, Retirement, Risk factors, Workload

Received: March 28, 2018 Accepted: May 17, 2018

Corresponding author: Yun Jeong Kim, MD, PhD

Department of Emergency Medicine, Kyungpook National University

School of Medicine, 680 Gukchaebosang-ro, Jung-gu, Daegu 41944,

Korea

E-mail: eumto@naver.com

This is an Open Access article distributed under the terms of the Creative Commons Attribution Non-Commercial License (http://creativecommons.org/licenses/bync/4.0/) which permits unrestricted non-commercial use, distribution, and reproduction in any medium, provided the original work is properly cited.

\section{INTRODUCTION}

Unlike regular retirement, early retirement is based on a voluntary decision to prematurely leave one's professional employment permanently. Although it may be positively associated with the beginning of a new project, most individuals are led to early retirement by the negative forces of employment mismatch due to work-related stress, burnout, and wage dissatisfaction $[1,2]$. 
Emergency physicians (EPs) have been reported to experience the highest levels of stress and burnout among medical specialists due to their constant exposure to a high-pressure working environment [3-6]. Prominent levels of work-related stress and burnout are known to contribute to early retirement among EPs [7].

Since the first generation of Korean EPs completed formal training in 1996, the working environment of emergency departments (EDs) has improved significantly [8]. There has been a decrease in night shifts and weekend/holiday shifts, while wages and workforce size have increased [8]. Despite these improvements, 2015 data revealed that only $70.6 \%$ of Korean EPs were working in an ED, which is a drop from $75.0 \%$ in 2010 $[8,9]$. The high early retirement rate among EPs is a significant issue with a potentially major negative impact on workforce management in the emergency medicine system. In other countries, in contrast, the attrition rates of EPs are lower than those of physicians in other medical specialties [10].

Studies have found that personal characteristics and extrinsic factors such as the working environment and wages contribute significantly to employees' voluntary early retirement [11-16]. However, it is necessary to identify other factors influencing the rising rate of early retirement among EPs despite steady improvements in the external working environment in emergency medicine.

Although an individual's intention to retire early may not necessarily lead to early retirement, this intention is closely related to actual behavior. For this reason, the intention to retire early has been adopted as a useful parameter in many studies [17]. Very few Korean studies have examined retirement rates among EPs or their intention to retire prematurely from their specialty. Therefore, we analyzed the factors associated with early retirement among EPs in Korea in order to identify measures that would promote their career longevity.

\section{METHODS}

We obtained approval from the Korean Society of Emergency Medicine, which conducts the Korean Emergency Physician Survey every 5 years. In response to increasing demand for emergency medicine and following the national policy regarding the advancement of the Korean emergency medical system, this survey was conducted to identify the current status of Korean EPs' working environment, and its results may contribute to policy-making and to improving working conditions.
The 2015 survey was based on the 2014 Longitudinal Study of Emergency Physicians, a 5-year survey by the American Academy of Emergency Medicine [18]. According to Herzberg's motivation-hygiene theory, relevant variables were grouped into the following 3 categories: personal characteristics, extrinsic factors such as the working environment, and intrinsic factors relating to medical professionalism [19]. Early retirement was defined as the act of voluntarily leaving emergency medicine practice before the preretirement age of 55 years.

Based on a previous study by Adams and Beehr [20] concerning early retirement, the item "Have you seriously considered early retirement from emergency medicine clinical practice?" was used to assess early retirement intention. This item was answered on a 5-point Likert scale, with a score of 4 or 5 indicating intention to retire early.

The survey was conducted over a 3-month period spanning from August 1 to October 31, 2015, using a paper-based questionnaire delivered 3 times or an electronic questionnaire delivered a maximum of 10 times via e-mail. For the electronic questionnaire, Adobe Acrobat ${ }^{\mathbb{R}}$ (Adobe Systems Inc., San Jose, (A, USA) was used to create an active file that could be filled out online. This file was subsequently posted on the Korean Society of Emergency Medicine website, as well as individually e-mailed to all registered members of the Korean Society of Emergency Medicine, comprising 1418 EPs. A total of 437 EPs participated in the survey. Of these, data from 29 who were over the age of 55 years or had retired from clinical practice at EDs, 27 who were on military service, and 4 who left multiple questionnaire items unanswered were excluded. Data from a total of 377 respondents were included in the analysis.

The participants completed the survey upon consenting to its purpose and intent detailed at the start of the survey. Considering that most respondents left 1-2 items unanswered, with a random distribution of unanswered items, missing values were statistically imputed for the analysis.

Statistical analysis was performed with SAS version 9.4 (SAS Institute Inc., Cary, NC, USA). To develop the nomogram, the nomogram function included in the $\mathrm{R}$ 3.3.2 rms package was used. The mean and standard deviation were calculated for continuous variables, and the $t$-test was used for comparisons between respondents with and without early retirement intention. For categorical variables, the chi-square test was performed. The significance level was set at $5 \%$. For the prediction model, a stepwise logistic regression analysis was performed with a $10 \%$ significance level to maximize the model's fitness. 
A nomogram was developed to facilitate the identification of weighted values pertaining to explanatory variables included in the prediction equation and to make the calculation of probability scores more straightforward.

\section{RESULTS}

The results of the analysis of early retirement intention according to personal characteristics are displayed in Table 1. Nearly half of the respondents expressed an intention to retire early. No differences in early retirement intention were found according to age, sex, or marital status. Duration of employment, health status, access priorities growth assistance responsibility (APGAR) score, and turnover history were associated with early retirement intention. Exercise, alcohol consumption, and smoking were not significantly associated with early retirement intention.

The results of the analysis of early retirement intention according to extrinsic factors are displayed in Table 2. EPs who were employed at academic hospitals showed a significantly lower rate of early retirement intention than their counterparts employed at non-academic hospitals. The mean hours worked per week and the mean number of night shifts in 4 weeks for EPs working in academic hospitals were not significantly different from their counterparts' values.

Early retirement intention decreased as the annual patient volume and ED safety increased. Early retirement intention was higher when there were 6 or more EPs available, and when the perception of workload intensity was higher than that of peers in other specialties. Regarding salary, early retirement intention was lower among those in the lower salary ranges, and higher among those in the highest salary range.

The results of the analysis of early retirement intention according to intrinsic factors are displayed in Table 3 . Early retirement intention decreased as satisfaction with the specialty (emergency medicine) and its outlook increased. Verbal abuse and receiving slanderous reviews from patients and their family members were significantly associated with a higher rate of early retirement intention. Aptitude for the specialty was not found to have a significant association with early retirement intention. Nevertheless, early retirement intention was lower among the respondents who stated that emergency medicine was an exciting field or that it provided opportunities to discover new medical technology and knowledge compared to those who provided negative responses. Early retirement in-
Table 1. EPs' early retirement intention by general characteristics

\begin{tabular}{|c|c|c|c|c|c|}
\hline \multirow[b]{2}{*}{ Variable } & \multirow[b]{2}{*}{ EPs (n) } & \multicolumn{2}{|c|}{ Retirement intention } & \multirow[b]{2}{*}{$\chi^{2}$} & \multirow[b]{2}{*}{$\begin{array}{c}p- \\
\text { value }\end{array}$} \\
\hline & & $\begin{array}{c}\text { Yes } \\
(n=181)\end{array}$ & $\begin{array}{c}\text { No } \\
(n=196)\end{array}$ & & \\
\hline Age (y) & & & & 2.99 & 0.22 \\
\hline$<40$ & 184 & $80(43.5)$ & $104(56.5)$ & & \\
\hline $40-49$ & 167 & $87(52.1)$ & $80(47.9)$ & & \\
\hline$\geq 50$ & 26 & 14 (53.8) & $12(46.2)$ & & \\
\hline Mean \pm SD & $40.75 \pm 5.14$ & & & & \\
\hline Sex & & & & 0.03 & 0.87 \\
\hline Male & 328 & $158(48.2)$ & $170(51.8)$ & & \\
\hline Female & 49 & $23(46.9)$ & $26(53.1)$ & & \\
\hline Marital status & & & & 3.54 & 0.06 \\
\hline Married & 336 & $167(49.7)$ & $169(50.3)$ & & \\
\hline Single & 41 & $14(34.1)$ & $27(65.9)$ & & \\
\hline Years working in ED & & & & 8.63 & 0.03 \\
\hline$<5$ & 94 & 31 (36.9) & $53(63.1)$ & & \\
\hline $5-9$ & 156 & $75(48.1)$ & 81 (51.9) & & \\
\hline $10-14$ & 84 & $50(59.5)$ & $34(40.5)$ & & \\
\hline$\geq 15$ & 53 & $25(47.2)$ & $28(52.8)$ & & \\
\hline Mean $\pm S D$ & $8.7 \pm 5.3$ & & & & \\
\hline Self-rated health & & & & 11.03 & 0.004 \\
\hline Good & 104 & 41 (39.4) & $63(60.6)$ & & \\
\hline Medium & 189 & $87(46.0)$ & $102(54.0)$ & & \\
\hline Poor & 84 & $53(63.1)$ & $31(36.9)$ & & \\
\hline Regular exercise & & & & 2.32 & 0.13 \\
\hline Yes & 168 & 88 (52.4) & $80(47.6)$ & & \\
\hline No & 209 & $93(44.5)$ & $116(55.5)$ & & \\
\hline Alcohol consumption & & & & 3.41 & 0.06 \\
\hline No & 35 & $22(62.9)$ & $13(37.1)$ & & \\
\hline Yes & 342 & $159(46.5)$ & $183(53.5)$ & & \\
\hline Smoking & & & & 0.06 & 0.80 \\
\hline Yes & 106 & $52(49.1)$ & $54(50.9)$ & & \\
\hline No & 271 & $129(47.6)$ & 142 (52.4) & & \\
\hline APGAR score ${ }^{1}$ & & & & 8.07 & 0.02 \\
\hline$\leq 5$ & 222 & $120(54.1)$ & 102 (45.9) & & \\
\hline $6-8$ & 114 & $46(40.4)$ & 68 (59.6) & & \\
\hline $9-10$ & 41 & $15(36.6)$ & $26(63.4)$ & & \\
\hline Mean \pm SD & $5.12 \pm 2.32$ & & & & \\
\hline Turnover history & & & & 39.26 & $<0.001$ \\
\hline Yes & 95 & 72 (75.8) & $23(24.2)$ & & \\
\hline No & 282 & $109(38.7)$ & $173(61.3)$ & & \\
\hline
\end{tabular}

Values are presented as number (\%).

EPs, emergency physicians; SD, standard deviation; ED, emergency department; APGAR, access priorities growth assistance responsibility.

${ }^{1} A n$ assessment of overall health of adults and families. 
Table 2. EPs' early retirement intention by extrinsic factors

\begin{tabular}{|c|c|c|c|c|c|}
\hline \multirow[b]{2}{*}{ Variable } & \multirow[b]{2}{*}{ EPs } & \multicolumn{2}{|c|}{ Retirement intention } & \multirow[b]{2}{*}{$\chi^{2}$} & \multirow[b]{2}{*}{$\begin{array}{c}p- \\
\text { value }\end{array}$} \\
\hline & & $\begin{array}{c}\text { Yes } \\
(n=181)\end{array}$ & $\begin{array}{c}\text { No } \\
(n=196)\end{array}$ & & \\
\hline Hospital type & & & & 7.04 & 0.008 \\
\hline Academic & 183 & $75(41.0)$ & $108(59.0)$ & & \\
\hline Non-academic & 194 & $106(54.6)$ & $88(45.4)$ & & \\
\hline No. of EPs in ED & & & & 5.54 & 0.02 \\
\hline$\leq 2$ & 34 & $21(61.8)$ & $13(38.2)$ & & \\
\hline $3-5$ & 229 & $114(49.8)$ & $115(50.2)$ & & \\
\hline$\geq 6$ & 114 & $46(40.4)$ & $68(59.6)$ & & \\
\hline Mean \pm SD & $4.3 \pm 1.9$ & & & & \\
\hline Annual patient volume & & & & 7.73 & 0.01 \\
\hline$<20000$ & 106 & $59(55.7)$ & $47(44.3)$ & & \\
\hline $20000-40000$ & 128 & $61(47.7)$ & $67(52.3)$ & & \\
\hline $40000-60000$ & 83 & $41(49.4)$ & $42(50.6)$ & & \\
\hline$>60000$ & 60 & $20(33.3)$ & $40(66.7)$ & & \\
\hline Salary $\left(10^{4} \mathrm{KRW} / \mathrm{mo}\right)$ & & & & 5.21 & 0.04 \\
\hline$<700$ & 70 & 30 (42.9) & $40(57.1)$ & & \\
\hline 700-999 & 104 & $44(42.3)$ & $60(57.7)$ & & \\
\hline 000-1299 & 83 & $42(50.6)$ & $41(49.4)$ & & \\
\hline $1300-1499$ & 77 & $39(50.6)$ & $38(49.4)$ & & \\
\hline$\geq 1500$ & 43 & $26(60.5)$ & $17(39.5)$ & & \\
\hline Hours worked (hr/wk) & & & & 6.70 & 0.08 \\
\hline$<39$ & 73 & $37(50.7)$ & $36(49.3)$ & & \\
\hline $40-49$ & 166 & $89(53.6)$ & $77(46.4)$ & & \\
\hline $50-59$ & 74 & $27(36.5)$ & $47(63.5)$ & & \\
\hline$\geq 60$ & 64 & $28(43.8)$ & $36(56.3)$ & & \\
\hline Mean $\pm S D$ & $51.6 \pm 14$ & & & & \\
\hline Night shifts in 4 wk & & & & 0.97 & 0.62 \\
\hline 0 & 32 & $14(43.8)$ & $18(56.3)$ & & \\
\hline $1-4$ & 59 & $29(49.2)$ & $30(50.8)$ & & \\
\hline $5-8$ & 220 & $104(47.3)$ & $116(52.3)$ & & \\
\hline$\geq 9$ & 66 & $35(53.0)$ & $31(47.0)$ & & \\
\hline Mean $\pm S D$ & $7.6 \pm 3.0$ & & & & \\
\hline \multicolumn{4}{|c|}{ Intensity of workload compared with other specialties } & 10.17 & 0.003 \\
\hline Normal & 165 & $67(40.6)$ & 98 (59.4) & & \\
\hline High & 160 & $80(50.0)$ & $80(50.0)$ & & \\
\hline Very high & 52 & $34(65.4)$ & $18(34.6)$ & & \\
\hline Safety in ED & & & & 20.72 & $<0.001$ \\
\hline Serious problem & 47 & 36 (76.6) & $11(23.4)$ & & \\
\hline Problem & 108 & 55 (50.9) & $53(49.1)$ & & \\
\hline Not a problem & 222 & $90(40.5)$ & 132 (59.5) & & \\
\hline
\end{tabular}

Values are presented as number or number (\%).

EPs, emergency physicians; ED, emergency department; SD, standard deviation.

tention was higher among the respondents who stated that the ED was heavily influenced by outside factors.
Table 3. EPs' early retirement intention by intrinsic factors

\begin{tabular}{|c|c|c|c|c|c|}
\hline \multirow[b]{2}{*}{ Variable } & \multirow[b]{2}{*}{ EPs } & \multicolumn{2}{|c|}{ Retirement intention } & \multirow[b]{2}{*}{$\chi^{2}$} & \multirow[b]{2}{*}{$p$-value } \\
\hline & & $\begin{array}{c}\text { Yes } \\
(n=181)\end{array}$ & $\begin{array}{c}\text { No } \\
(n=196)\end{array}$ & & \\
\hline \multicolumn{2}{|l|}{ Slanderous reviews } & & & 7.26 & 0.03 \\
\hline None (1) & 236 & $101(42.8)$ & $135(57.2)$ & & \\
\hline A few (2) & 72 & $39(54.2)$ & $33(45.8)$ & & \\
\hline Many $(3+4+5)$ & 69 & $41(59.4)$ & $28(40.6)$ & & \\
\hline \multicolumn{2}{|l|}{ Verbal abuse } & & & 10.76 & 0.003 \\
\hline None & 65 & $24(36.9)$ & $41(63.1)$ & & \\
\hline Medium & 94 & $42(44.7)$ & $52(55.3)$ & & \\
\hline High & 218 & $115(52.8)$ & $103(42.2)$ & & \\
\hline \multicolumn{2}{|l|}{ Satisfaction with specialty } & & & 23.00 & $<0.001$ \\
\hline Not satisfied & 110 & $73(66.4)$ & $37(33.6)$ & & \\
\hline Moderately satisfied & 107 & $49(45.8)$ & $58(54.2)$ & & \\
\hline Satisfied & 160 & 59 (36.9) & $101(63.1)$ & & \\
\hline \multicolumn{2}{|l|}{ Aptitude for the specialty } & & & 4.18 & 0.12 \\
\hline No fit & 255 & $118(46.3)$ & $137(53.7)$ & & \\
\hline Medium fit & 88 & $41(46.6)$ & $47(53.4)$ & & \\
\hline Good fit & 34 & $22(64.7)$ & $12(35.3)$ & & \\
\hline \multicolumn{2}{|l|}{ Satisfaction with EMS } & & & 11.37 & 0.003 \\
\hline Not satisfied & 207 & $115(55.6)$ & $92(44.4)$ & & \\
\hline Moderately satisfied & 140 & $52(37.1)$ & 88 (62.9) & & \\
\hline Satisfied & 30 & $14(46.7)$ & $16(53.3)$ & & \\
\hline \multicolumn{2}{|l|}{ Outlook of EM } & & & 35.43 & $<0.001$ \\
\hline Not satisfied & 88 & $66(75.0)$ & $22(25.0)$ & & \\
\hline Moderately satisfied & 141 & $62(44.0)$ & $79(56.0)$ & & \\
\hline Satisfied & 148 & $53(35.8)$ & $95(64.2)$ & & \\
\hline \multicolumn{3}{|c|}{ ED depending on external factors } & & 8.86 & 0.01 \\
\hline No & 51 & $19(37.3)$ & $32(62.7)$ & & \\
\hline Medium & 114 & $46(40.4)$ & $68(59.6)$ & & \\
\hline Yes & 212 & $116(54.7)$ & $96(45.3)$ & & \\
\hline \multicolumn{2}{|l|}{ Poor reception } & & & 3.73 & 0.15 \\
\hline No & 25 & $9(36.0)$ & $16(64.0)$ & & \\
\hline Medium & 88 & $37(42.0)$ & $51(58.0)$ & & \\
\hline Yes & 264 & $135(51.1)$ & $129(48.9)$ & & \\
\hline \multicolumn{2}{|l|}{ Exciting work } & & & 10.07 & 0.007 \\
\hline No & 18 & $15(83.3)$ & $3(16.7)$ & & \\
\hline Medium & 61 & $31(50.8)$ & $30(49.2)$ & & \\
\hline Yes & 298 & $135(45.3)$ & $163(54.7)$ & & \\
\hline \multicolumn{2}{|l|}{ New skills and medicine } & & & 8.12 & 0.02 \\
\hline No & 52 & $34(65.4)$ & $18(34.6)$ & & \\
\hline Medium & 159 & $76(47.8)$ & 83 (52.2) & & \\
\hline Yes & 166 & $71(42.8)$ & $95(57.2)$ & & \\
\hline
\end{tabular}

Values are presented as number or number (\%).

EPs, emergency physicians; EM, emergency medicine; EMS, emergency medical services; ED, emergency department.

The results of the logistic regression analysis of early retirement intention according to personal characteristics, extrinsic 
Table 4. Logistic regression analysis of early retirement intention

\begin{tabular}{lccccc}
\hline Variable & Estimate & SE & $\chi^{2}$ & $\boldsymbol{p}$-value & $\begin{array}{c}\mathbf{O R} \\
(\mathbf{9 5 \%} \mathbf{~ C I})\end{array}$ \\
\hline $\begin{array}{l}\text { Intercept } \\
\text { Age (y) }\end{array}$ & -6.83 & 1.16 & -34.58 & $<0.001$ & \\
$<40$ & 1.00 & & & & \\
$40-49$ & 0.8 & 0.26 & 9.62 & 0.002 & 2.24 \\
& & & & & $(1.34,3.72)$ \\
$\geq 50$ & 1.06 & 0.48 & 4.88 & 0.03 & 2.88 \\
& & & & & $(1.13,7.36)$
\end{tabular}

Hospital type

Academic 1.00

Non-academic

0.65

6.31

0.01

1.91

$(1.15,3.17)$

Self-rated health

0.29

(score: 1-5)

Intensity of workload

(score: 3-5)

ED safety

(score: 3-5)

Slanderous reviews

(score: $1-5$ )

0.34

$0.18 \quad 2.75$

0.10

1.41

$(1.04,1.91)$

1.34

$(0.95,1.90)$

$\begin{array}{lllll}0.49 & 0.18 & 7.51 & 0.006 & 1.64\end{array}$

$(1.15,2.33)$

$\begin{array}{lllll}0.24 & 0.12 & 3.81 & 0.05 & 1.27\end{array}$

$(1.00,1.61)$

Satisfaction with specialty

5

4

3

2

$$
1
$$

1.00

1.01

0.49

4.29

0.04

2.75

$(1.06,7.17)$

$\begin{array}{lllll}0.78 & 0.44 & 3.2 & 0.07 & 2.19\end{array}$

$(0.93,5.18)$

$\begin{array}{lllll}0.87 & 0.44 & 3.89 & 0.05 & 2.38\end{array}$

$(1.01,5.61)$

1.5

0.54

7.72

$0.006 \quad 4.49$

$(1.56,12.97)$

Outlook of EM

$\begin{array}{lllllc}\text { Medium (3+4+5) } & 1.00 & & & & \\ \text { Not satisfied (2) } & 1.08 & 0.34 & 5.14 & 0.02 & 2.94 \\ & & & & & (1.52,5.69) \\ \text { Very not satisfied (1) } & 1.56 & 0.69 & 10.17 & 0.001 & \begin{array}{c}4.78 \\ \end{array} \\ & & & & & (1.24,18.46)\end{array}$

$\mathrm{SE}$, standard error; OR, odds ratio; $\mathrm{Cl}$, confidence interval; $\mathrm{ED}$, emergency department; EM, emergency medicine.

factors, and intrinsic factors are displayed in Table 4. Regarding personal characteristics, risk factors contributing to early retirement intention were age and health status. Contributing extrinsic risk factors were hospital type, workload intensity relative to other specialties, and ED safety. Intrinsic factors contributing to early retirement intention included slanderous reviews from patients and family members, and specialty satisfaction level and its outlook. The discriminatory power of the early retirement intention model was 0.762 , and the following retirement prediction equation was obtained using the logistic regression model:

Linear predictor $(\mathrm{LP})=-6.83+0.80 \mathrm{Age} 1+1.06 \mathrm{Age} 2+0.65 \mathrm{HT}+$ $0.29 \mathrm{HS}+0.34 \mathrm{IW}+0.49 \mathrm{SA}+0.24 \mathrm{SR}+0.87 \mathrm{SS} 1+0.78 \mathrm{SS} 2+1.01 \mathrm{SS} 3+$ $1.50 \mathrm{SS} 4+1.08 \mathrm{OL} 1+1.58 \mathrm{OL} 2$

Here, Age 1 is 1 or 0 depending on age (1: aged 40-49 years; 0 : other). Similarly, Age2 is 1 or 0 depending on age (1: aged 50 years and older; 0 : other). $\mathrm{HT}$ is either 1 or 0 depending on employment at an academic hospital (0: employed at an academic hospital; 1: other). HS represents health status, IW workload intensity, SA emergency room safety, and SR slanderous reviews. Additionally, SS1 to SS4 represent the level of satisfaction with emergency medicine as a specialty: SS1 is 1 when the satisfaction score is 2, and 0 when not; SS2 is 1 when the satisfaction score is 3 , and 0 when not; SS3 is 1 when the satisfaction score is 4, and 0 when not; and SS4 is 1 when the satisfaction score is 5, and 0 when not. Finally, OL1 and OL2 represent the level of satisfaction with the outlook of emergency medicine as a specialty: OL 1 is 1 for a response indicating dissatisfaction ( 2 points) and 0 otherwise; and OL2 is 1 for a response indicating severe dissatisfaction (1 point) and 0 otherwise. Upon calculating the LP score, the following equation was used to obtain the probability of early retirement intention.

$$
P(\mathrm{Y}=\text { early retirement intention })=1 /(1+\exp [-\mathrm{LP}])
$$

The nomogram for scoring the probability of early retirement intention obtained via the above equation is shown in Figure 1.

\section{DISCUSSION}

This was the first Korean study to examine factors influencing early retirement among EPs and to develop a model for predicting early retirement intention. We analyzed the causes of early retirement among EPs, including personal, extrinsic, and internal factors. The risk factors for early retirement among EPs include individual factors such as age and health status; extrinsic factors such as hospital type, perceived workload intensity, and emergency room safety; and intrinsic factors such as level of satisfaction with emergency medicine as a specialty and its outlook and slanderous reviews. Although some of these factors, such as age and hospital type, are outside of an individual's control, other factors such as health status, emergency room safety, working conditions, and slanderous reviews may be controlled through enhanced mutual re- 


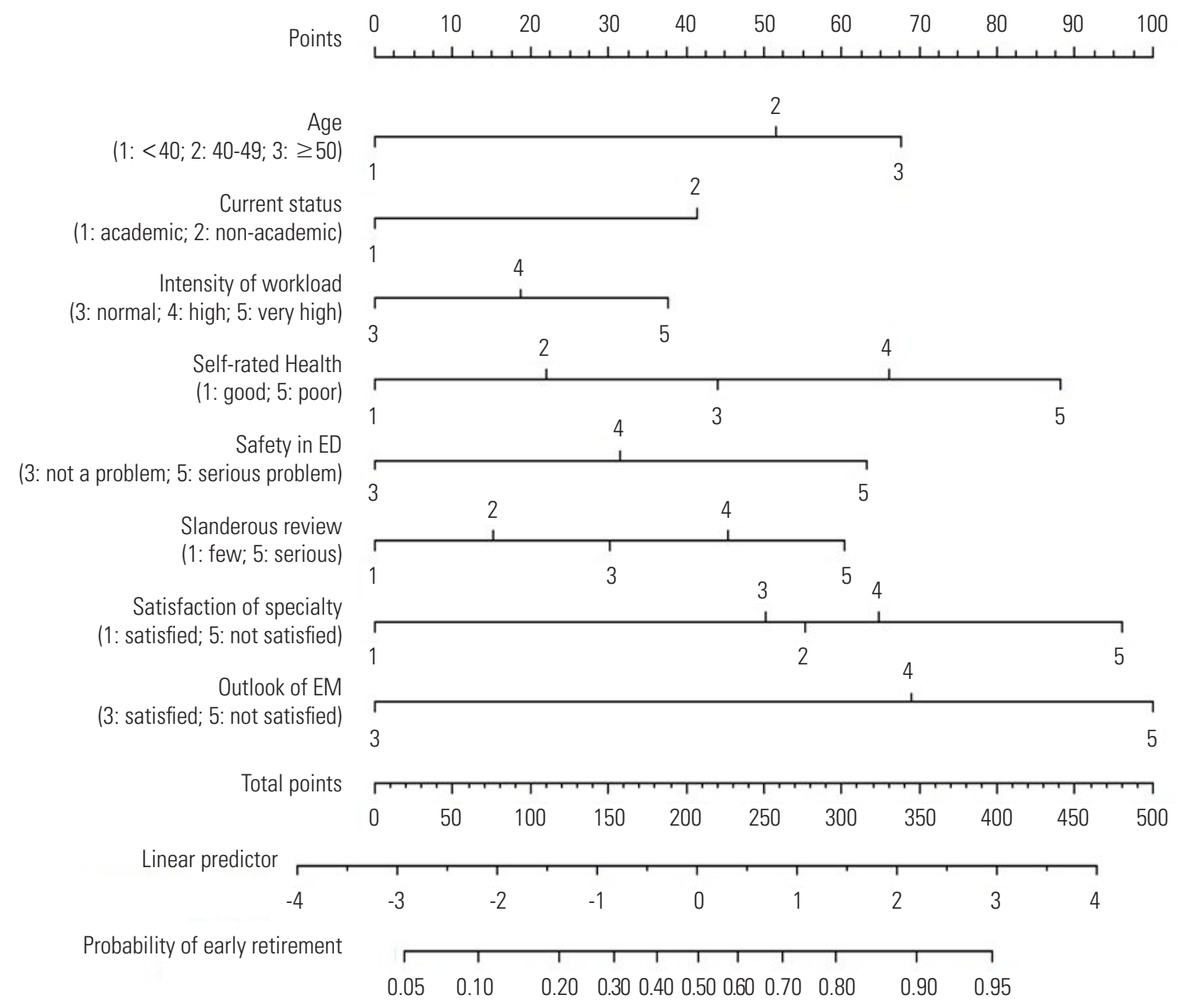

Figure 1. Nomogram for scoring the probability of early retirement intention. The instructions are as follows: Calculate the score for each factor using the points scale at the top and sum the points. Then, draw a straight vertical line from the total points to intersect the indicator of probability at the bottom, which will indicate the probability of early retirement intention. ED, emergency department; EM, emergency medicine.

spect between patients and doctors; furthermore, satisfaction with the specialty and its outlook may also be influenced by promoting medical professionalism. The equation developed in the present study enabled the prediction of early retirement intention by calculating scores based on the weighted value of each risk factor in a nomogram. An example of score calculation using nomogram is described in Figure S1 and Table S1. To reduce early retirement rates and promote career longevity among EPs, selective improvements designed to target this high-risk group are critical.

Early retirement not only involves the disruption of a professional career, in which EPs have invested heavily in terms of both time and cost, but also leads to decreased morale and increased workload for peers. In addition, increasing costs to recruit and secure newly trained replacements can have a signif- icant negative impact on workforce management, which is a critical issue in emergency medicine at both the hospital and national levels. For these reasons, it is important to promote career longevity. A total of 1418 EPs have qualified in Korea as of July 2015. Of these, only 999 (70.6\%) worked in EDs in 2015 [9]. The approximate $30.0 \%$ attrition rate, although it includes normal retirements, deaths, and leaves of absence, mostly reflects early retirements and the subsequent opening of private practices $[8,12]$. Additionally, at $48.0 \%$, a high proportion of the respondents in the current study expressed early retirement intention. In comparison, $87.0 \%$ of EPs in the US are currently practicing, and the annual attrition rate of $1.5 \%$ is lower than that in other medical specialties [10].

Early retirement is associated with various individual factors. Burnout, stress, and the patient-doctor relationship have been 
reported to play an important role in early retirement among physicians [21,22].

Among personal factors, age, duration of employment as an $E P$, health status, and history of turnover were associated with early retirement intention. The mean age of the respondents was 40.8 years, and early retirement intention was very high among those in their early 40s, who typically have 10-14 years of professional experience as EPs. In comparison, the mean age of EPs currently employed at emergency rooms across the US was 44 years; and the most common timing of early retirement from emergency medicine practice was 20 years following the acquisition of the relevant qualification, indicating a much longer tenure in the emergency room than among their Korean counterparts [10].

Work-related stress and burnout among EPs are significantly associated with extrinsic factors such as shift work, night shifts, work-family conflict, the fishbowl environment, and violent situations [12-16]. In particular, night shifts and shift work are known to contribute greatly to early retirement [4]. Among these extrinsic factors, working hours have decreased in Korea to an average of 51.6 hours weekly, and the number of night shifts to 7.6 nights monthly [8]. In relation to these improvements, the present study did not find working hours or night shifts to be significantly associated with early retirement intention. Early retirement intention was higher among those in the highest salary range in the present study, but this finding appears to be linked to the hospital type, given that specialists employed at non-academic hospitals typically receive very generous compensation. The mean number of EPs working at regional emergency medical centers, where the workload intensity of EPs is higher, was 7.7, which was significantly higher than at non-regional emergency centers [9]. The higher rate of intention to retire early observed in EDs with 6 or more EPs seemed to be linked to this condition. In a similar manner, annual patient volume appeared to be related to workload intensity, but further investigation is needed to identify the correlation between those factors. Early retirement intention was greater among EPs employed at non-academic hospitals than among their counterparts employed at academic hospitals, and this finding was similar to previous research $[12,23]$. Emergency room safety protocols pertaining to unforeseen violence and damage/destruction of property, as well as workload intensity relative to other specialties, were found to be strongly associated with early retirement intention. Violence against medical staff by patients, family members, and indi- viduals under the influence of alcohol, as well as damage/destruction of property, have also been found to greatly contribute to work-related stress in other studies $[24,25]$.

Intrinsic factors influencing early retirement included professional attitude and satisfaction with the specialty, the outlook of emergency medicine, research, and training in new skills and knowledge, and these were analyzed and studied from the viewpoint of medical professionalism. In the present study, it was found that early retirement intention decreased as the level of satisfaction increased regarding emergency medicine as a specialty and its outlook. Early retirement intention also increased as slanderous reviews increased. According to Hann et al. [26], the factors influencing job satisfaction include extrinsic factors such as monetary compensation, working conditions, and workload intensity, as well as intrinsic factors such as sense of achievement, social role and status, and autonomy; furthermore, enhancing intrinsic factors plays a significant role in increasing one's sense of career achievement. According to Cydulka and Korte [27], a diverse patient group and opportunities to participate in training and administrative tasks, in addition to generous compensation and improved working conditions, are important contributors to a robust sense of career achievement. Other studies have reported that difficulties in the patient-doctor relationship could cause burnout, contributing to early retirement [22]. In conclusion, the intrinsic factors identified as contributors to early retirement in the above studies appear to be associated with medical professionalism. While it is important for physicians to provide patient care according to the principles of optimizing patient welfare and autonomy, it is equally important for them to maintain professional competence by keeping up with new medical knowledge and skills. We also confirmed that an important current threat to physicians and healthcare is distrust and excessive demands, which subsequently contribute to early retirement. For this reason, a patient-doctor partnership based on mutual respect, where public trust and rights are well-balanced, should form the core of medical professionalism [28-30].

Finally, we performed a stepwise logistic regression analysis to identify a prediction model for early retirement intention. This was followed by the development of a nomogram to identify the weighted values of explanatory variables included in the prediction equation and to facilitate the calculation of prediction scores. Using the prediction equation, each respondent's early retirement intention probability score was calcu- 
lated. Eight risk factors were included in the prediction equation. The probability of early retirement intention increased among physicians with higher age, in non-academic hospitals with a greater workload relative to other specialties, poorer health status, more slanderous reviews, and lower level of satisfaction with emergency medicine as a specialty or its outlook. Of these, the weighted values of the intrinsic factors were the largest. Therefore, according to the prediction equation in the present study, the intrinsic factors had a greater influence on early retirement intention than did the extrinsic factors known to be important causes of early retirement among physicians. Extrinsic factors such as night shifts, shift work, and compensation had a small influence on early retirement intention, whereas the level of satisfaction with emergency medicine as a specialty and its outlook, as well as mutual respect between patient and doctor, were significantly associated with early retirement intention.

This study has several limitations. First, because the study only focused on EPs, the prediction equation may not be suitable for application among doctors in other medical specialties. Second, although depression, burnout, satisfaction with work-life balance, and administrative tasks apart from patient treatment may also be associated with early retirement among EPs, their associations with early retirement intention could not be verified because these parameters were not included in the survey questionnaire. Finally, because the data used in the study were gathered via a self-report and crosssectional survey questionnaire, each respondent's mental and physical state at the time of completion may have influenced the accuracy of responses. The possibility of sampling error by enthusiastic respondents or missing data errors due to unanswered items cannot be ruled out. EPs who did not have a verified mailing address or did not respond for any reason may either have already been retired or were working under poor conditions that would induce dissatisfaction.

In conclusion, to reduce the rate of early retirement among EPs and to promote career longevity, not only extrinsic factors, but also intrinsic factors such as professional identity and mutual patient-doctor respect must be enhanced. Doing so will foster a healthy balance between professional duties and rights.

\section{ACKNOWLEDGEMENTS}

This work was supported by Kyungpook National University and Korean Society of Emergency Medicine.

\section{CONFLICT OF INTEREST}

The authors have no conflicts of interest associated with the material presented in this paper.

\section{SUPPLEMENTAL MATERIALS}

Supplementary Material 1: Table $\mathrm{S} 1$ is available at https:// www.jpmph.org/.

Supplementary Material 2: Figure S1 is available at https:// www.jpmph.org/.

\section{ORCID}

Jaemyeong Shin http://orcid.org/0000-0002-1549-1805

Yun Jeong Kim http://orcid.org/0000-0002-7906-9734

Jong Kun Kim http://orcid.org/0000-0002-0583-4368

\section{REFERENCES}

1. Jovanovic B. Job matching and the theory of turnover. J Polit Econ 1979;87(5 Pt 1):972-990.

2. Landon BE, Aseltine R Jr, Shaul JA, Miller Y, Auerbach BA, Cleary PD. Evolving dissatisfaction among primary care physicians. Am J Manag Care 2002;8(10):890-901.

3. Shanafelt TD, Boone S, Tan L, Dyrbye LN, Sotile W, Satele D, et al. Burnout and satisfaction with work-life balance among US physicians relative to the general US population. Arch Intern Med 2012;172(18):1377-1385.

4. Smith-Coggins R, Broderick KB, Marco CA. Night shifts in emergency medicine: the american board of emergency medicine longitudinal study of emergency physicians. J Emerg Med 2014; 47(3):372-378.

5. Estryn-Behar M, Doppia MA, Guetarni K, Fry C, Machet G, Pelloux $\mathrm{P}$, et al. Emergency physicians accumulate more stress factors than other physicians-results from the French SESMAT study. Emerg Med J 2011;28(5):397-410.

6. Keller KL, Koenig WJ. Sources of stress and satisfaction in emergency practice. J Emerg Med 1989;7(3):293-299.

7. Lee YK, Lee CC, Chen CC, Wong CH, Su YC. High risk of 'failure' among emergency physicians compared with other specialists: a nationwide cohort study. Emerg Med J 2013;30(8):620622.

8. Lee HM, Cho KH, Yang HJ, Kim IB, Lee KJ, Han SB, et al. 2015 Korean Society of Emergency Physician Survey (KEPS): major 
changes from 2010 to 2015. J Korean Soc Emerg Med 2016; 27(5 Suppl):25-40 (Korean).

9. National Emergency Medical Center. Annual statistical report of emergency medicine 2015 [cited 2016 Sep 25]. Available from: http://www.e-gen.or.kr/nemc/statistics_annual_report. do (Korean).

10. Ginde AA, Sullivan AF, Camargo CA Jr. Attrition from emergency medicine clinical practice in the United States. Ann Emerg Med 2010;56(2):166-171.

11. Feldman DC. The decision to retire early: a review and conceptualization. Acad Manag Rev 1994;19(2):285-311.

12. Nho WY, Kim JK. Emergency physician turnover in private clinics. J Korean Soc Emerg Med 2011;22(6):716-727 (Korean).

13. Doan-Wiggins L, Zun L, Cooper MA, Meyers DL, Chen EH. Practice satisfaction, occupational stress, and attrition of emergency physicians. Wellness Task Force, Illinois College of Emergency Physicians. Acad Emerg Med 1995;2(6):556-563.

14. Lloyd S, Streiner D, Hahn E, Shannon S. Development of the emergency physician job satisfaction measurement instrument. Am J Emerg Med 1994;12(1):1-10.

15. Tziner A, Rabenu E, Radomski R, Belkin A. Work stress and turnover intentions among hospital physicians: the mediating role of burnout and work satisfaction. J Work Organ Psychol 2015; 31(3):207-213.

16. Heponiemi T, Kouvonen A, Vänskä J, Halila H, Sinervo T, Kivimäki $\mathrm{M}$, et al. Health, psychosocial factors and retirement intentions among Finnish physicians. Occup Med (Lond) 2008;58(6): 406-412.

17. Brown SP, Peterson RA. Antecedents and consequences of salesperson job satisfaction: meta-analysis and assessment of causal effects. J Mark Res 1993;30(1):63-77.

18. American Board of Emergency Medicine. Longitudinal Study of Emergency Physicians; 2014 [cited 2018 May 19]. Available from: https://www.abem.org/public/docs/default-source/default-document-library/2014-five-year-survey.pdf?sfvrsn $=0$.

19. Sachau DA. Resurrecting the motivation-hygiene theory: herzberg and the positive psychology movement. Hum Resour Dev Rev 2007;6(4):377-393.
20. Adams GA, Beehr TA. Turnover and retirement: a comparison of their similarities and differences. Pers Psychol 1998;51(3): 643-665.

21. Moreno-Jiménez B, Gálvez-Herrer M, Rodríguez-Carvajal R, Sanz Vergel Al. A study of physicians' intention to quit: the role of burnout, commitment and difficult doctor-patient interactions. Psicothema 2012;24(2):263-270.

22. Hahn SR. Physical symptoms and physician-experienced difficulty in the physician-patient relationship. Ann Intern Med 2001;134(9 Pt 2):897-904.

23. Kim JK, Kim YJ, Seo KS, Ryoo HW, Kam S, Park JY, et al. Job stress, job satisfaction and occupational commitment among Korean emergency physicians. J Korean Soc Emerg Med 2010; 21(2):246-258 (Korean).

24. Chen KC, Hsieh WH, Hu SC, Lai PF. A survey of the perception of well-being among emergency physicians in Taiwan. Ci Ji Yi Xue Za Zhi 2017;29(1):30-36.

25. Verelst S, Moonen PJ, Desruelles D, Gillet JB. Emergency department visits due to alcohol intoxication: characteristics of patients and impact on the emergency room. Alcohol Alcohol 2012;47(4):433-438.

26. Hann M, Reeves D, Sibbald B. Relationships between job satisfaction, intentions to leave family practice and actually leaving among family physicians in England. Eur J Public Health 2011;21(4):499-503.

27. Cydulka RK, Korte R. Career satisfaction in emergency medicine: the ABEM Longitudinal Study of Emergency Physicians. Ann Emerg Med 2008;51(6):714-722.

28. Project of the ABIM Foundation; ACP-ASIM Foundation; European Federation of Internal Medicine. Medical professionalism in the new millennium: a physician charter. Ann Intern Med 2002;136(3):243-246.

29. Cohen JJ, Cruess S, Davidson C. Alliance between society and medicine: the public's stake in medical professionalism. JAMA 2007;298(6):670-673.

30. Working Party of the Royal College of Physicians. Doctors in society. Medical professionalism in a changing world. Clin Med (Lond) 2005;5(6 Suppl 1):S5-S40. 